\title{
RESULTADOS DEL EXAMEN DE UBICACIÓN DE MATEMÁTICAS (EXUMAT 2.0) EN LA FACULTAD DE INGENIERÍA ENSENADA DE LA UABC
}

\author{
Results of the EXUMAT Exam in Mathematics taking \\ at the Autonomous University of Baja California (UABC) \\ School of Engeneering
}

Jana-Juracy Soares-López ${ }^{1}$

Everardo Inzunza-González ${ }^{2}$

Pablo Rousseau-Figueroa ${ }^{3}$

\begin{abstract}
Resumen: Se presenta un análisis comparativo entre el número de alumnos reprobados en la materia de Matemáticas I (Cálculo Diferencial) que es el primer curso de matemáticas que los estudiantes llevan en la Facultad de Ingeniería Ensenada de la Universidad Autónoma de Baja California (UABC), con los resultados del examen de ubicación EXUMAT el cual determina el nivel educativo real con el que un estudiante que ha salido de bachillerato, llega a la Universidad. Los resultados indican que un mayor porcentaje de estudiantes aprueban la materia, en comparación con lo esperado de acuerdo al EXUMAT. En el presente trabajo se discuten las posibilidades de esta discrepancia.
\end{abstract}

Palabras clave: Matemáticas. Universidad. Reprobados.

\begin{abstract}
A comparative analysis between the number of students who failed to get a pass grade in the Mathematics I Course (Differential Calculus), the first mathematics course the students take at the Engineering Faculty of the Autonomous University of Baja California (UABC), and the results of the EXUMAT exam, which determines the real educational level in which a student who finishes $12^{\text {th }}$ grade, reaches the University. The results indicate that a greater percentage of the students pass the Mathematics I course in comparison to what is expected according to EXUMAT. The possibilities of this discrepancy are discussed in the present analysis.
\end{abstract}

Keywords: Mathematics. University. Failed students.

\footnotetext{
${ }^{1}$ Maestría en Ciencias de la Tierra. Docente en matemáticas, Universidad Autónoma de Baja California (UABC). México.juracy@uabc.mx

${ }^{2}$ Maestría en Ciencias en Electrónica y Telecomunicaciones. Docente, Curso de Circuitos Digitales III, Universidad Autónoma de Baja California (UABC). einzunza@uabc.mx

${ }^{3}$ Maestría en Ingeniería Hidráulica. Docente en Hidráulica I, Universidad Autónoma de Baja California (UABC). México.pablorousseau@uabc.mx
}

${ }^{1}$ Universidad Autónoma de Baja California

Campus Ensenada - Km. 103 Carret

Tij-Ens Ensenada, B. C.

22860 México

121

Ciência \&̊ Educação, v. 15, n. 1, p. 121-8, 2009 
Soares-López, J-J.; Inzunza-González, E.; Rousseau-Figueroa, P.

\section{Introducción}

Los resultados de PISA (Programme for International Student Asessment) de la Organización para la Cooperación y el Desarrollo Económico (OCDE) de 2003, muestran que la media de desempeño en la escala global de matemáticas para nuestro país es de 385, por debajo del promedio OCDE de 500, ocupando el lugar número 37 de los quarenta países participantes en ese año. Si a lo anterior se le agrega el hecho que de 31 estados, Baja California ocupa el lugar \#17 con 384 puntos en la evaluación de las secundarias, pudiera significar que nuestro estado pierde competitividad desde la Educación Básica.

En la Facultad de Ingeniería de la Universidad Autónoma de Baja California (UABC), en general, el índice de reprobación ha ido en incremento; desde 1992-I se ha dado seguimiento a lo anterior por parte de la entonces Coordinadora del área de matemáticas de la Facultad de Facultad de Ingeniería Ensenada (FIE) mediante la recopilación de datos desde 1992-I utilizando los registros escolares de los exámenes ordinarios, extraordinarios y de regularización de los três primeros semestres en las materias del área de matemáticas.

En 1993, el M. C. Fortunato Espinoza, analiza las evaluaciones efectuadas por los profesores de matemáticas de la Facultad de Ingeniería en las materias de Matemáticas I (1. semestre), Cálculo de varias variables (3. semestre) y Ecuaciones Diferenciales (3. semestre) mostrando a partir de los resultados de las evaluaciones, que éstas "dependen principalmente de los maestros, destacándose dos tendencias opuestas: 1) maestros con los que aprueban casi todos los alumnos y 2) maestros con los que pocos alumnos aprueban" (ESPINOZA, 1993). En los resultados de su trabajo, asocia los grupos con las notas más altas en las três materias antes mencionadas, con un mismo profesor X1.

Dicho trabajo presenta más datos fundamentando las tendencias existentes y concluyendo que se debe efectuar un análisis de los métodos de evaluación de los cursos, de manera que dependan de los contenidos y no de los profesores (ESPINOZA, 1993).

En octubre de 2004, la FIE recibió una invitación por parte del Instituto de Investigación y Desarrollo Educativo de la de la misma Universidad, para participar en la aplicación del Examen de Ubicación de Matemáticas EXUMAT, en su versión 2.0 (BACKHOFF, IBARRA, ROSAS, 1994) a los estudiantes de primer semestre.

El EXUMAT fue diseñado para evaluar las competencias de los alumnos de acuerdo al currículum oficial de los niveles educativos.

Dicho examen tiene como objetivo «ubicar en su dimensión real de conocimientos y habilidades en Matemáticas, a los estudiantes, de manera que los mismos tengan una medida del nivel con que enfrentarán a las matemáticas universitarias; por otra parte, a los profesores les permite hacer un tratamiento de los contenidos acorde a ese nivel de los estudiantes» (BACKHOFF, IBARRA, ROSAS, 1994, p. 3).

Un grupo de profesores de la Facultad de Facultad de Ciencias de la UABC, tomando los libros de texto de matemáticas en los diferentes grados educativos ubicaron los niveles de conocimientos de acuerdo a lo que consideraron fuesen básicos y típicos de cada grado (BACKHOFF, IBARRA, ROSAS, comunicación personal, 1994), proponiendo las siguientes áreas nodales de conocimientos: 
Nivel Primario- Números naturales, fraccionarios, decimales; longitudes, áreas y volúmenes; capacidad, peso, tiempo, cuerpos geométricos, figuras geométricas, tratamiento de la información, predicción y azar.

Nivel Secundario- Los números naturales y decimales y sus operaciones; sistemas de numeración; números con signo; números primos y compuestos; porcentaje; iniciación al lenguaje algebráico; preálgebra; probabilidad; ecuaciones y sistemas de ecuaciones; operaciones con monomios y polinomios; ángulos entre paralelas y una secante; equivalencia de figuras y cálculo de áreas; sólidos; raíz cuadrada y cálculo aproximado; fracciones algebráicas; productos notables y factorización; triángulos y cuadriláteros; círculo; el teorema de Pitágoras; elementos de trigonometría.

Nivel preuniversitario- El sistema de los números reales; productos notables; factorización; leyes de los exponentes; ecuaciones racionales lineales con una variable; inecuaciones lineales con una variable; ecuaciones cuadráticas; ángulos y su medición en grados y radianes; la circunferencia unitaria y las funciones trigonométricas definidas en esta figura; triángulos oblicuángulos; leyes de los senos y cosenos; regresión y correlación lineal; conceptos básicos de coordenadas rectangulares; la línea recta, la circunferencia, la parábola, la elipse; distribuciones de probabilidad; funciones; límite de una función; derivación de funciones algebraicas, derivadas de funciones trascendentes, máximos y mínimos de funciones, fórmulas fundamentales de integración, integración utilizando cambio de variable, la integral definida.

Para llevar a cabo la confección del examen, se tomó en cuenta los objetivos a lograr por cada grado o cada año de cada tipo de nivel. Los reactivos permiten dar respuestas abiertas de diferentes tipos como numérica, textual y cerrada. La calificación de las preguntas es 1 si es correcta y $\mathbf{0}$ en caso de ser incorrecta.

El presente trabajo relaciona los resultados del examen EXUMAT aplicado a estudiantes de la Facultad de Ingeniería Ensenada de primer semestre, con el número de reprobados en la materia de matemáticas I (cálculo diferencial) que es el primer curso de matemáticas que reciben dichos estudiantes.

\section{Metodología}

\section{Examen EXUMAT}

Backhoff, Ibarra e Rosas (1994) fundamentaron el examen en la Teoría de Respuestas al ítem, con el modelo de dos parámetros (de discriminación y de dificultad). Para administrar el EXUMAT 2.0 diseñaron la interfaz computarizada llamada "Sistema de Exámenes Adapta- 
tivos (SEA)" capaz de presentar los reactivos tanto en forma secuencial como en forma adaptativa y de calificar preguntas de respuesta abierta de diferentes tipos: numéricas, algebráicas, textuales y de opción múltiple 4 .

Los conceptos matemáticos han sido ordenados de menor a mayor complejidad, con un total de 219 reactivos, de los cuales para primaria corresponden un total de 66 reactivos, para secundaria 92 reactivos y finalmente para bachillerato se tienen 61 reactivos. Las respuestas varían en modalidad, pudiendo ser numéricas y enteras, textuales, numéricas y decimales, de opción múltiple etc.

En la Tabla 1 se muestran ejemplos de preguntas y respuestas del examen EXUMAT en su versión 2.0.

El examen computarizado fue aplicado en el mes de octubre del semestre 2004-II, que es aproximadamente a mitad del semestre, atendiendo a un total de 178 alumnos de primer semestre de las carreras de ingeniería (o tronco común) quienes participaron en este examen.

La calificación de las respuestas es $\mathbf{1}$ si es correcta y $\mathbf{0}$ en caso de ser incorrecta.

Tabla 1. Tipo de preguntas y respuestas del EXUMAT 2.0.

\begin{tabular}{|c|c|c|}
\hline Tipo de pregunta & Ejemplo & Respuesta \\
\hline $\begin{array}{l}\text { Respuesta numérica, } \\
\text { entera }\end{array}$ & $\begin{array}{l}\text { Un excursionista hizo un recorrido de cinco } \\
\text { días; el primer día caminó } 39 \mathrm{~km} \text {, el } \\
\text { segundo } 43 \mathrm{~km} \text {, el tercero } 27 \mathrm{~km} \text {, el cuarto } \\
19 \mathrm{~km} \text { y el quinto día recorrió } 32 \mathrm{~km} \text {. } \\
\text { ¿cuántos km recorrió en los primeros três } \\
\text { días? }\end{array}$ & 109 \\
\hline $\begin{array}{l}\text { Respuesta numérica, } \\
\text { entera }\end{array}$ & $\begin{array}{l}\text { Resolver el sistema de ecuaciones } \\
\text { siguiente: } 2 x+y=5 ; 3 x+y=8\end{array}$ & $x=3, y=-1$ \\
\hline $\begin{array}{l}\text { Respuesta numérica } \\
\text { con decimales }\end{array}$ & $\begin{array}{l}\text { Se compraron dos máquinas. Si una costó } \$ \\
68.85 \text { y otra } \$ 57.65 \text {, ¿cuánto se gastó en } \\
\text { total? }\end{array}$ & $\$ 126.5$ \\
\hline Respuesta textual & $\begin{array}{l}\text { Escribir con palabras en pesos y centavos } \$ \\
352.75\end{array}$ & $\begin{array}{l}\text { Trescientos cincuenta y dos } \\
\text { pesos con setenta y cinco } \\
\text { centavos. }\end{array}$ \\
\hline
\end{tabular}

\section{Resultados}

En la Figura 1 se muestra a manera de histogramas, los resultados arrojados por el examen EXUMAT, donde se puede observar un comportamiento simétrico centrado en promedio, entre las calificaciones de 5 y 6.

${ }^{4}$ Leer el artículo completo para una explicación detallada del Programa. 
Resultados de la aplicación del exame ...

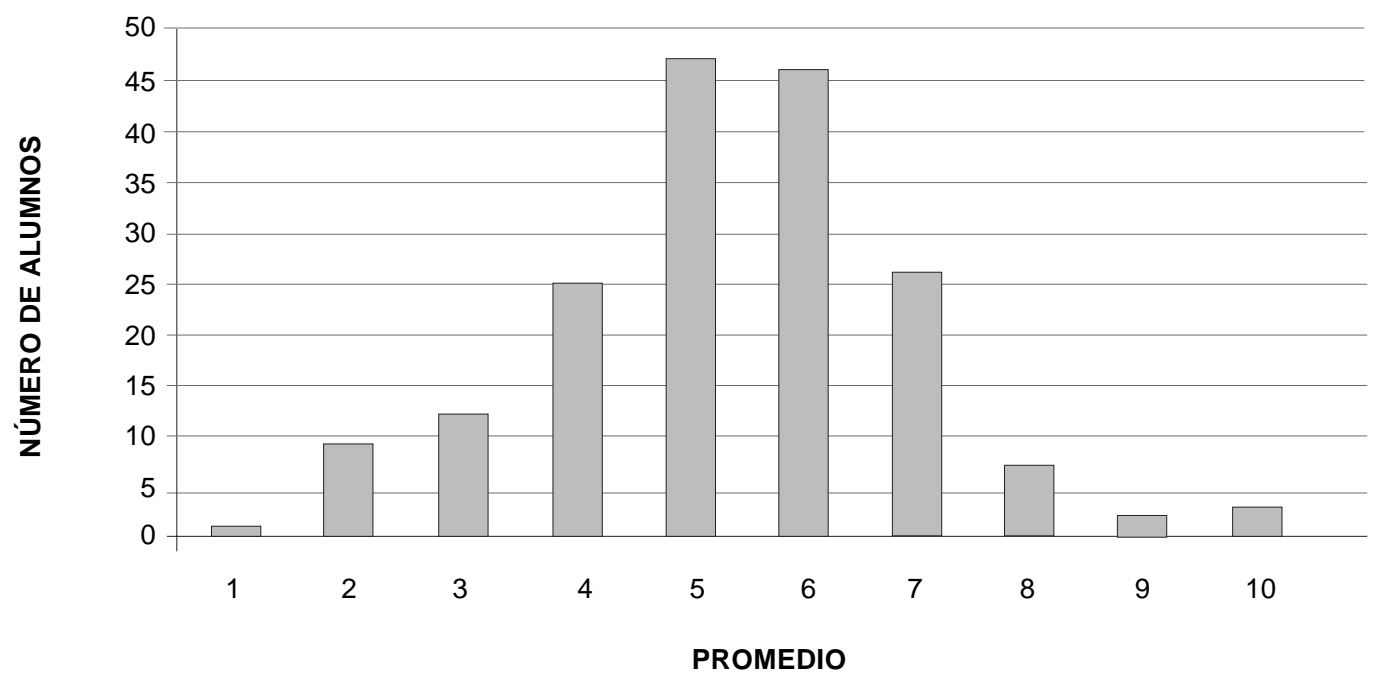

Figura 1. Promedio de calificaciones de 178 alumnos que presentaron el examen EXUMAT en el año 2004-II.

Aún con ese nivel, los alumnos han aprobado el bachillerato y han sido aceptados por la Universidad Autónoma de Baja California mediante un examen de admisión.

En la Figura 2 se muestra una comparación de resultados del EXUMAT entre los dos sistemas principales de donde los alumnos provienen: DGTI y COBACH. Se observa que el $53 \%$ de los alumnos provenientes del sistema DGTI se encuentran académicamente ubicados en el nivel de 3. secundaria y 1 . de bachillerato conjuntamente, mientras que para el sistema Cobach, un total de 68\% se ubica en 1. y 2. semestre de bachillerato. Cabe mencionar que el bachillerato en México, consiste de 6 semestres. La Figura 3 muestra el desempeño del resto de los alumnos provenientes de otros bachilleratos incorporados.

Durante el semestre de 2004-II, nueve profesores tuvieron a su cargo los 16 grupos existentes que llevaron la materia de Matemáticas I (Cálculo Diferencial); algunos profesores tuvieron un grupo y otros hasta três grupos. La media del porcentaje de aprobados es menor del $50 \%$ (sin tomar en cuenta el número de alumnos por grupo). Un grupo consistente únicamente de alumnos repetidores de la materia, obtuvo un bajo índice de aprobación (Tabla 2, grupo B). La Tabla 2 muestra el alto porcentaje que el maestro "F" tiene en los grupos a su cargo, mientras que le profesor "J" presenta, para el mismo curso, el menor porcentaje de aprobados, lo anterior, sin tomar en cuenta el grupo especial de repetidores. 
Soares-López, J-J.; Inzunza-González, E.; Rousseau-Figueroa, P.

DGIT (63)
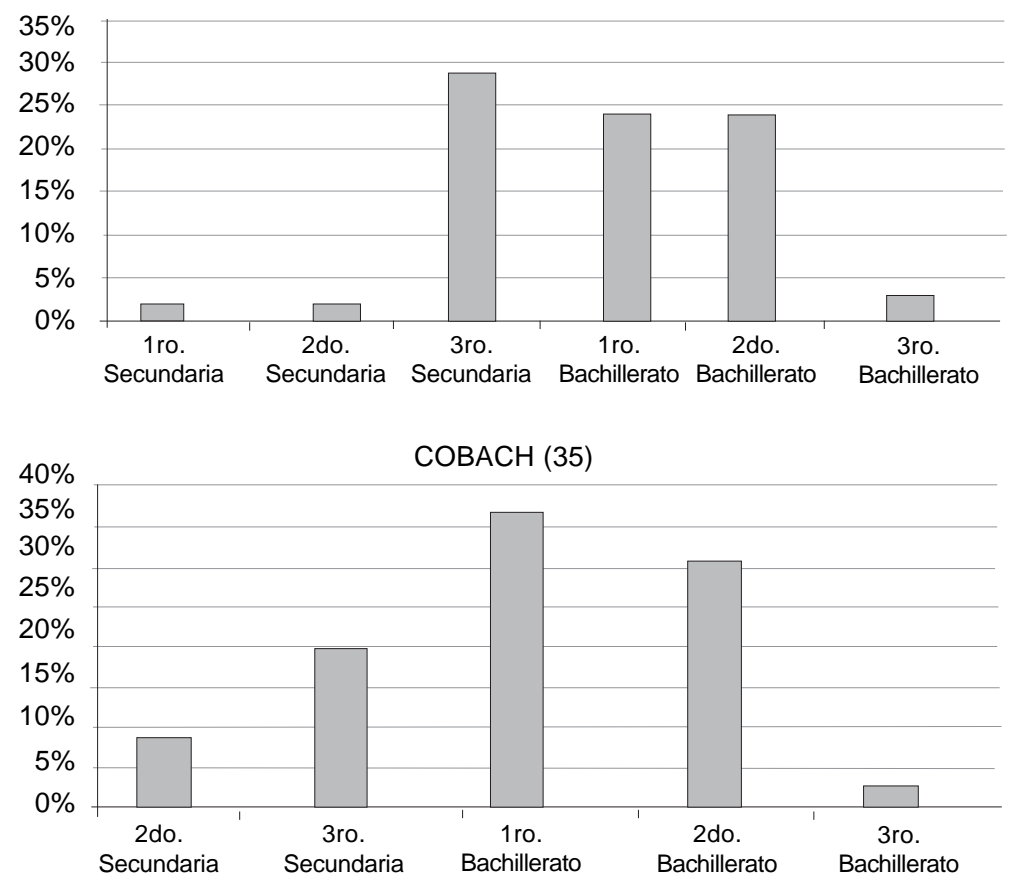

Figura 2. Porcentaje de alumnos de los principales sistemas educativos DGTI y COBACH ubicados en los diferentes niveles educativos de acuerdo al examen EXUMAT.

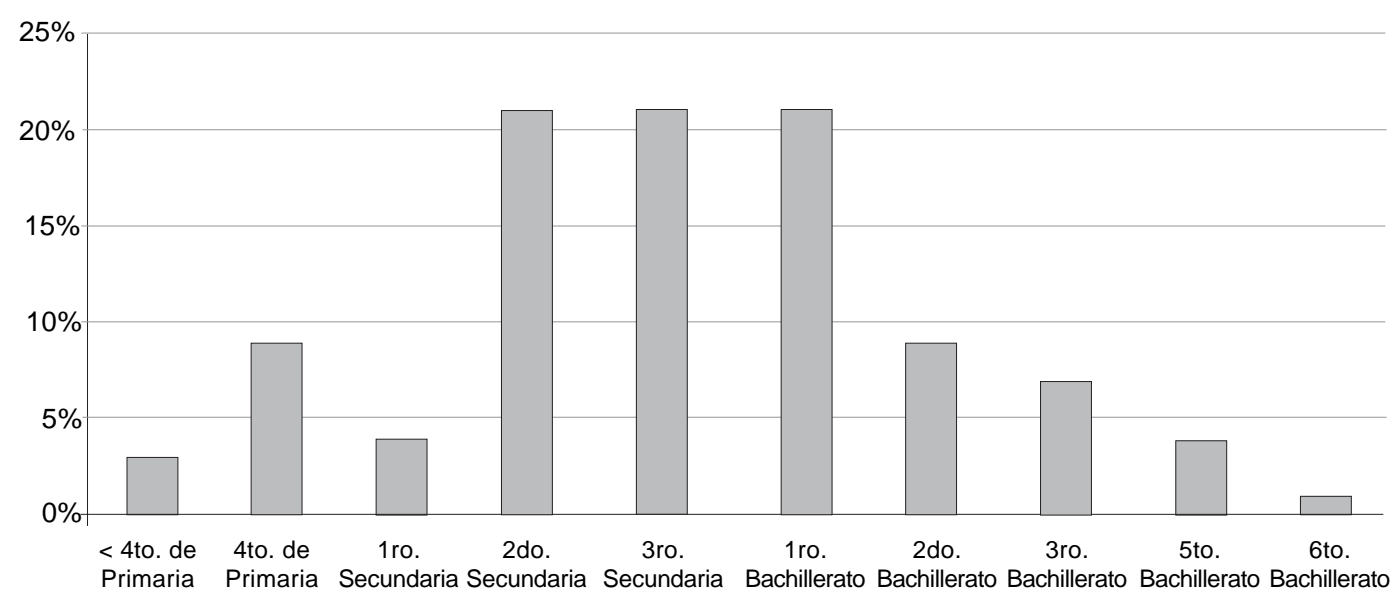

BACHILLERATOS RESTANTES

Figura 3. Porcentaje de alumnos del resto de los bachilleratos o preparatorias incorporados a alguno de los sistemas principales. 
Tabla 2. Clasificación por profesor y número total de alumnos aprobados en sus grupos, siendo el $64.3 \%$ el mayor y el $13.6 \%$ el menor.

\begin{tabular}{lrc}
\hline \multicolumn{4}{c}{$\begin{array}{c}\text { Relación entre profesor y número de aprobados } \\
\text { en la materia de Matemáticas I }\end{array}$} \\
\multicolumn{2}{c}{ en la Facultad de Ingeniería Ensenada de la UABC } \\
\hline Profesor & \% de aprobados & Número alumnos \\
\hline A & 39 & 41 \\
B & 13.6 & 22 \\
C & 35 & 20 \\
D & 55.87 & 51 \\
E & 50 & 18 \\
F & 64.3 & 95 \\
G & 52.5 & 40 \\
H & 62.8 & 35 \\
I & 50.69 & 69 \\
J & 20 & 70 \\
\hline
\end{tabular}

\section{Conclusiones}

Aún con el nivel de conocimientos encontrado, los alumnos cursan y aprueban el bachillerato y son aceptados por la Universidad mediante un examen que sólo es para ubicar al alumno, pero no para seleccionar a los mejores, lo cual dificulta el trabajo del profesor universitario de Matemáticas I ya que además de enseñar nuevos conceptos, debe darse a la tarea de intentar solucionar las deficiencias con las que el estudiante llega.

El 21.34\% aprueba el EXUMAT, y de éstos, la mayoría lo hace con una calificación de 6, lo cual no significa que tengan los conocimientos que se requieren para cursar exitosamente sus primeros semestres en la Facultad de Ingeniería Ensenada; sin embargo al observar la Tabla 2, puede notarse que en la mayoría de los grupos de Matemáticas I, el porcentaje de alumnos aprobados es notablemente mayor que lo mostrado por los resultados del EXUMAT ya que se esperaría que los alumnos que no cuentan con las bases necesarias para cursar exitosamente Matemáticas I, reprobaran la materia.

Dicha disparidad pudiese significar, ya sea:

1. Que algunas de las preguntas aplicadas en el examen de conocimientos no tiene incidencia sobre los contenidos temáticos del curso de Matemáticas I en la Facultad de Ingeniería.

2. Que algunos de los profesores de la Facultad de Ingeniería pudieran estar sobrevaluando al alumno.

\section{Discusión}

Aún cuando los investigadores -autores del programa EXUMAT- establecen como uno de sus objetivos que el profesor lleve a cabo un tratamiento de los contenidos acorde al 
Soares-López, J-J.; Inzunza-González, E.; Rousseau-Figueroa, P.

nivel de los estudiantes, la realidad en el aula es que los grupos son heterogéneos, con aproximadamente 45 estudiantes por materia y además, se deben cumplir tiempos y el $80 \%$ del contenido temático como mínimo, de acuerdo con el plan de clase y horas establecidas para desarrollar dicho contenido, sin incluir la existencia de los días festivos; esto aunado a la falta de responsabilidad y compromiso de los estudiantes con su propio aprendizaje, dificulta la enseñanza ya que el maestro debe cubrir el curso y los errores permanecen.

Se propone:

a) La inclusión de un examen de selección propio y adecuado para el ingreso de los alumnos a la FIE, que cumpla con estándares de universidades internacionales en las áreas de ingeniería tomando en cuenta los programas vigentes de la Secretaría de Educación Pública, previa aprobación de un curso propedéutico o de apoyo educativo como requisito indispensable para el ingreso a esta Facultad.

b) Un adecuado número de horas para realizar la labor de tutorías así como un número adecuado de estudiantes por tutor, lo cual coadyuvaría a su proceso de aprendizaje.

c) La supervisión de los padres hacia los hijos en la realización de sus tareas así como la retroalimentación periódica hacia estos mentores respecto al desempeño académico de sus hijos, pudiendo ser ésta accesada por internet o entregada personalmente.

d) La realización de un curso propedéutico con una duración mínima de un semestre, mismo que de cualquier manera se considera perdido para aquellos estudiantes de bajo rendimiento, o con conocimientos previos deficientes.

e) Elaboración de exámenes colegiados para homologar los criterios de evaluación entre los profesores de la Facultad de Ingeniería y calificados por profesores diferentes a los titulares de la materia.

\section{Referências}

BACKHOFF, E.; IBARRA, M.A.; ROSAS, M. Versión computarizada del examen de habilidades y conocimientos básicos. In: CONGRESO INTERNACIONAL DE PSICOLOGÍA APLICADA, 23., 1994, Madrid. Anais?... Madrid, 1994.

ESPINOZA, F. Evaluación. In: CONGRESO NACIONAL DE LA ASOCIACIÓN NACIONAL DE PROFESORES DE MATEMÁTICAS. MEMORIAS, 12., 1993, Querétaro, México. Anais... México, 1993. p. 232-5.

MINISTERIO DE EDUCACIÓN Y CIENCIA. Aprender para el mundo de mañana (2003). Resumen de Resultados PISA 2003. Disponível em: <http://www.ince.mec.es/ pub/pubintn.htm>. Acesso em: 29 abr. 2009.

Artigo recebido em maio de 2008 e aceito em novembro de 2008 